\title{
Dry Eye Due To Milletus Diabetes
}

\author{
Rizka Febriya Moestafa \\ Faculty of Medicine, University of Mataram, Indonesia \\ rizkafebriya02@gmail.com
}

Article Information

Accepted : 05 Nov 2021

Submitted:09 Nov 2021

Online Publish : 20 Nov

2021

\begin{abstract}
Eye dry syndrome or dry eye is a multifactorial disorder that causes reduced tear fluid, causing discomfort to the eye. Several risk factors that cause dry eye such as history of medication, meibomian gland dysfunction, age, gender, systemic disease and immune disorders (Mark, et al., 2021). Diabetes mellitus is one of the risk factors for dry eyes. The International Dry Eye Workshop II (DEWS) divides dry eyes into two parts based on etiopathological criteria, namely due to lack of tear production or Aqueous Tear Deficiency (ATD) and excessive evaporation or Evaporative Tear Deficiency (ETD). Prevalence This article aims to review Diabetes Miletus, one of the risk factors for dry eye. The source search was carried out on the online portal of the publication of the journal Nation Center for Biotechnology Information / NCBI, Google Scholar with the keywords "Eye Dry Syndrome, Diabetes Milletus, Eye Dry treatment".
\end{abstract}

Keywords : Dry Eye, Treatment Eye Dry, Diabetes Milletus 


\section{Introduction}

Diabetes Milletus is a chronic metabolic disease caused by an increase in blood sugar levels due to a decrease in insulin levels. According to the Diabetes Federation, Diabetes mellitus is a global health problem, around 415 million people in the world aged 20 to 79 years suffer from diabetes milletus (Goyal \& Jialal, 2018). Chronic diabetes mellitus causes various organ system complications, including retinopathy, nephropathy, neuropathy, and heart disease. Diabetes milletus is classified into type 1 and type 2. Complications of this disease cause disruption of the virtual water layer, namely Dry Eyes.

Dry eye is a multifactorial disease that requires treatment that can overcome the symptoms that arise. The symptoms caused include pain, red eyes, decreased vision (Lee, Kabat, Bacharach, Karpecki, \& Luchs, 2020). Some of the risk factors for dry eyes such as gender, age, vitamin A deficiency, and metabolic diseases. Dry eye disease is more common in women than men. Patients with diabetes mellitus who experience dry eyes are around $15-33 \%$ at the age of 65 years.

\section{Research methods}

The writing of this article uses various sources from scientific journals. The source search was conducted on the online portal of the publication of the journal Nation Center for Biotechnology Information/ NCBI, Google Scholar with the keywords "Eye Dry Syndrome, Diabetes Mellitus, Eye Dry treatment".

\section{Result and Discussion Definitions}

Diabetes Miletus (DM) is a metabolic disease characterized by hyperglycemia due to decreased insulin levels. Diabetes Mellitus is classified into type 1 and type 2 (Sapra, Bhandari, \& Wilhite Hughes, 2021). Uncontrolled hyperglycemia causes several organs to experience dysfunction, especially the eyes, kidneys, heart and blood vessels which can cause many complications such as neuropathy, stroke, and peripheral vascular disease (Aljarousha, Badarudin, \& Azemin, 2016). DM is a risk factor for dry eye.

Dry Eyes or Eye Dry Syndrome is a condition where tears are reduced so that they cannot lubricate the surface of the eye. The causes of dry eyes are multifactorial, characterized by conjunctival hyperemia, eye thickening, itching, burning sensation in the eyes, and often accompanied by decreased vision (Mark et al., 2021). This is accompanied by tear hyperosmolarity and inflammation of the ocular surface. The surface of the eye consists of the cornea, conjunctiva, lacrimal glands, meibomian glands (Messmer, 2015). Some of the factors that cause dry eyes are age, gender, antihistamines, drug history, diabetes mellitus, pregnancy, and vitamin A deficiency (Messmer, 2015) 


\section{Classification}

The International Dry Eye Workshop II (DEWS) divides dry eye classification into two parts based on etiopathological criteria, the mechanism is due to lack of tear production or Aqueous Tear Deficiency (ATD) and excessive evaporation or Evaporative Tear Deficiency (ETD) (Shimazaki, 2018). Dry Eyes Aqueous Tear Deficiency (ATD) is caused by failure of lacrimal tear secretion due to acinar lacrimal gland dysfunction or decreased volume of tear secretion causing hyperosmolarity due to normal evaporation. ATD is grouped into two, namely dry eye Sjogren's syndrome and dry eye, not Sjogren's syndrome. Sjogren's syndrome is an autoimmune disease that attacks the lacrimal gland, salivary glands, and several other organs. While nonSjogren's syndrome is dry eye associated with age, diabetes mellitus, AIDS.

Evaporative Tear Deficiency (ETD) occurs due to loss of tears on the surface of the eye, while the lacrimation glands function normally. This condition can be influenced by intrinsic factors (eyelid structure) such as meibomian gland dysfunction and extrinsic factors (eye surface disease) such as vitamin-A deficiency, use of contact lenses, and use of topical medications (Craig et al., 2017)

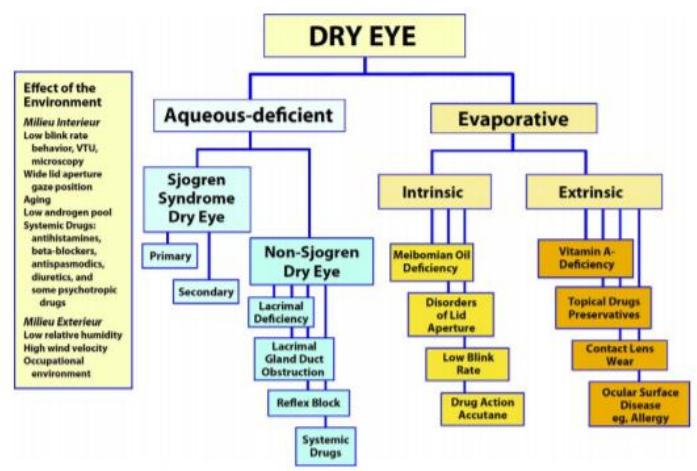

Figure 1.

Classification of a dry eye according to DEWS (Craig et al., 2017)

\section{Epidemiology}

Dry eye disease occurs at all ages from children to the elderly, and from various economic backgrounds (Le Trung et al., 2021). The prevalence of dry eye in people with diabetes mellitus is higher than in people without diabetes mellitus. Patients with diabetes mellitus who experience dry eyes are around $15-33 \%$ at the age of 65 years and are more common in women than men. $20 \%$ of dry eye cases occur in type 2 diabetes mellitus patients (Zhang, Zhao, Deng, Sun, \& Wang, 2016). The research was also conducted by (Zou et al., 2018), there were $17.5 \%$ of dry eye events in people with diabetes mellitus. 


\section{Rizka Febriya Moestafa / KESANS}

Dry Eye Due To Milletus Diabetes

\section{Etiology}

Dry eye is caused by disruption of the tear film. The tear film consists of lacrimal gland dysfunction. The lacrimal gland functions to maintain the tear film and keep the eye lubricated. In patients with diabetes mellitus, hyperglycemia occurs in the blood which affects the function of the lacrimal gland as a lubricant that causes dry eyes (Zhang et al., 2016)

\section{Pathophysiology}

Lack of tear production or Aqueous Tear Deficiency (ATD) and excessive evaporation or Evaporative Tear Deficiency (ETD) cause tear hyperosmolarity (Shimazaki, 2018). Hyperosmolarity can occur by activating a cascade of inflammatory mediators on the ocular surface (IL-1, TNF-, and matrix metalloproteinase (MMP9)) and these mediators are released into tears so that the ocular surface epithelium is damaged (Stepp et al., 2018). Meibomian gland dysfunction is the most common cause of dry eye. Meibomian gland dysfunction occurs due to obstruction of the glandular ducts and inhibits the secretion of the meibomian glands thereby facilitating bacterial growth. This increases the activation of enzymes that produce free fat that causes inflammation in the eye (Nebbioso et al., 2017). Decreased tear flow is also caused by failure of the lacrimal gland and increased evaporation of tear fluid. This condition causes tear film instability due to the release of inflammatory mediators so that the eye surface becomes damaged (Mark, et al., 2021).

Dry eyes in diabetes mellitus sufferers are caused by reduced tear production due to neuropathy (Najafi, Malek, Valojerdi, Khamseh, \& Aghaei, 2015). Neuropathy is caused by chronic high levels of glucose in the blood, which affects the work of the nerves that control the secretion of the lacrimal gland, damaging the eye film, which produces moisturizers to keep the eyes lubricated. When the sensitivity of the cornea is no longer properly lubricated it causes corneal cells to become damaged and expose free nerve endings. Free nerve endings cause symptoms of dry eye in DM patients (Zhang et al., 2016)

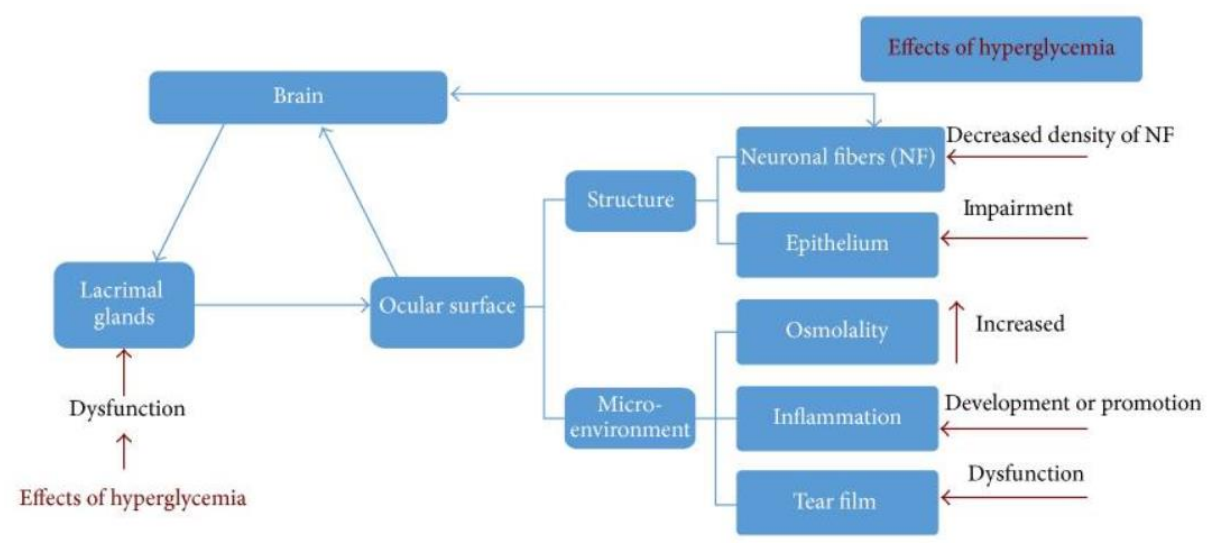


Figure 2.

Mechanism of dry eye due to hyperglycemia (Zhang et al., 2016)

\section{Clinical Manifestations}

Symptoms vary from mild to severe such as burning, stinging, itching, red eyes and can cause decreased vision and pain in severe cases. People with dry eyes also find it difficult to open their eyes when they wake up and symptoms get worse with exposure to wind.

\section{Management}

Of dry eye treatment varies depending on the severity of the patient and etiopathology (Mayer, 2020). Disorders of the tear film consisting of mucin, aqueous, and lipids cause dry eyes. Some of the topical drugs that can be given include diquafosol, rebamipide, gefarnate, ecabet sodium. Topical diaquafasol $3 \%$ and rebamipide $2 \%$ are the most widely available in the market and are used as an option for treating dry eye disease (Şimşek, Doğru, Kojima, \& Tsubota, 2018)

Protecting the surface of the eye can use autologous serum eye drops consisting of essential tear components and reducing pain, burning with anti-inflammatory such as corticosteroids (Zhang et al., 2016). In cases of meibomian gland dysfunction, the goal of treatment is to improve the flow of meibomian secretions and reduce exposure to antibiotics. Warm compresses to widen the orifices of the meibomian glands, soaps and scrubs to clean debris and bacterial colonies, and massage on the eyelids to facilitate thickened meibomian secretions. Lipiflow is a thermodynamic therapy for blocked meibomian glands, this disposable device is placed on the eyelids and distributes heat to the glands so that meibomian secretion occurs (Şimşek et al., 2018). In addition, educating patients to avoid factors that can aggravate complaints such as exposure to wind, sun exposure and eyelid hygiene (Mayer, 2020).

Corticosteroids are not given to patients with diabetes mellitus because there are side effects such as cataracts, bacterial and fungal infections, so non-corticosteroid antiinflammatory drugs are more commonly used. In addition, the administration of autologous serum needs to be considered in patients with diabetes mellitus because of the risk of causing bacterial growth. Patients with diabetes mellitus are educated on insulin therapy to reduce high glucose levels, change their lifestyle with regular exercise, diet, and avoid anxiety. Based on the results of the study, patients with diabetes mellitus with dry eye disorders can be given gene and cell therapy. 


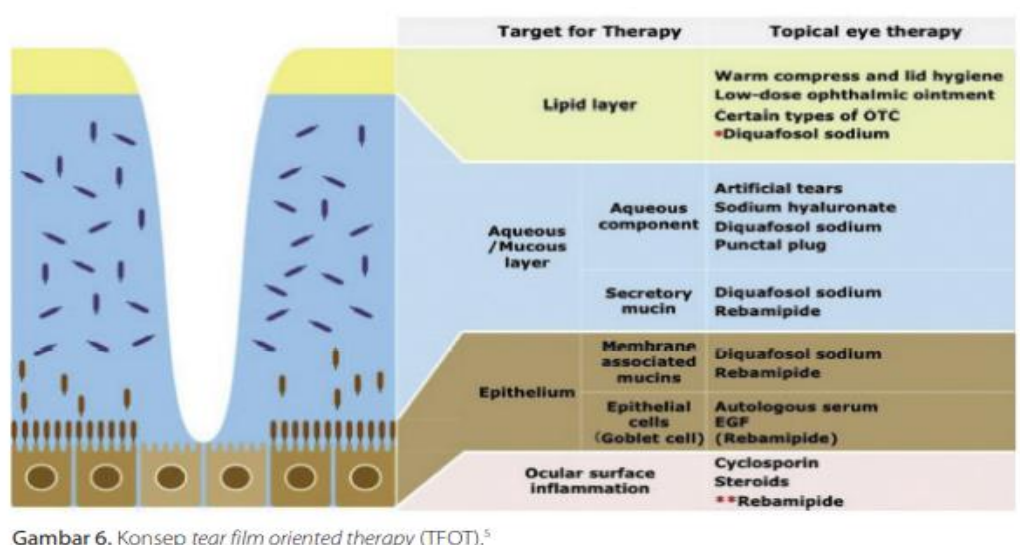

Figure 3.

Dry Eye Treatment Options (Şimşek et al., 2018)

\section{Conclusion}

Diabetes milletus is a metabolic disease characterized by an increase in blood glucose due to decreased insulin levels. Diabetes milletus is one of the risk factors for dry eye. Dry eye is multifactorial disease characterized by red eye, burning, pain, and decreased vision. The International Dry Eye Workshop II (DEWS) divides dry eye classification into two parts based on etiopathological criteria, the mechanism is due to lack of tear production or Aqueous Tear Deficiency (ATD) and the state of excessive evaporation or Evaporative Tear Deficiency (ETD). Patients with diabetes milletus are educated on insulin therapy to reduce high glucose levels. 


\section{REFERENCE}

Aljarousha, Mohammed, Badarudin, Noor Ezailina, \& Azemin, Mohd Zulfaezal Che. (2016). Comparison of dry eye parameters between diabetics and non-diabetics in district of Kuantan, Pahang. The Malaysian Journal of Medical Sciences: MJMS, 23(3), 72 .

Craig, Jennifer P., Nichols, Kelly K., Akpek, Esen K., Caffery, Barbara, Dua, Harminder S., Joo, Choun Ki, Liu, Zuguo, Nelson, J. Daniel, Nichols, Jason J., \& Tsubota, Kazuo. (2017). TFOS DEWS II definition and classification report. The Ocular Surface, 15(3), 276-283.

Goyal, Rajeev, \& Jialal, Ishwarlal. (2018). Diabetes mellitus type 2.

Le Trung, Nguyen, Toan, Pham Quoc, Le Viet Thang, Nguyen Dinh Ngan, Thang, Nguyen Chien, Van Cuong, Nguyen, Van Dam, Nguyen, Anh, Hoang Tuan, Hang, Vo Thi, Trung, Nguyen Kien, \& Do Nhu Binh, Vu Anh Tuan. (2021). The Relationship Between Dry Eye in Adults with Indications for Kidney Transplantation and Influence Factors. Clinical Ophthalmology (Auckland, NZ), $15,4327$.

Lee, Bridgitte Shen, Kabat, Alan G., Bacharach, Jason, Karpecki, Paul, \& Luchs, Jodi. (2020). Managing dry eye disease and facilitating realistic patient expectations: a review and appraisal of current therapies. Clinical Ophthalmology (Auckland, NZ), $14,119$.

Messmer, Elisabeth M. (2015). The pathophysiology, diagnosis, and treatment of dry eye disease. Deutsches Ärzteblatt International, 112(5), 71.

Najafi, Laily, Malek, Mojtaba, Valojerdi, Ameneh Ebrahim, Khamseh, Mohammad E., \& Aghaei, Hossein. (2015). Dry eye disease in type 2 diabetes mellitus; comparison of the tear osmolarity test with other common diagnostic tests: a diagnostic accuracy study using STARD standard. Journal of Diabetes \& Metabolic Disorders, 14(1), 1-8.

Nebbioso, Marcella, Del Regno, Paola, Gharbiya, Magda, Sacchetti, Marta, Plateroti, Rocco, \& Lambiase, Alessandro. (2017). Analysis of the pathogenic factors and management of dry eye in ocular surface disorders. International Journal of Molecular Sciences, 18(8), 1764.

Sapra, Amit, Bhandari, Priyanka, \& Wilhite Hughes, A. (2021). Diabetes Mellitus (Nursing).

Shimazaki, Jun. (2018). Definition and diagnostic criteria of dry eye disease: historical overview and future directions. Investigative Ophthalmology \& Visual Science, 59(14), DES7-DES12. 
Şimşek, Cem, Doğru, Murat, Kojima, Takashi, \& Tsubota, Kazuo. (2018). Current management and treatment of dry eye disease. Turkish Journal of Ophthalmology, 48(6), 309.

Stepp, Mary Ann, Pal-Ghosh, Sonali, Tadvalkar, Gauri, Williams, Alexa, Pflugfelder, Stephen C., \& de Paiva, Cintia S. (2018). Reduced intraepithelial corneal nerve density and sensitivity accompany desiccating stress and aging in C57BL/6 mice. Experimental Eye Research, 169, 91-98.

Zhang, Xinyuan, Zhao, Lin, Deng, Shijing, Sun, Xuguang, \& Wang, Ningli. (2016). Dry eye syndrome in patients with diabetes mellitus: prevalence, etiology, and clinical characteristics. Journal of Ophthalmology, 2016.

Zou, Xinrong, Lu, Lina, Xu, Yi, Zhu, Jianfeng, He, Jiangnan, Zhang, Bo, \& Zou, Haidong. (2018). Prevalence and clinical characteristics of dry eye disease in community-based type 2 diabetic patients: the Beixinjing eye study. $B M C$ Ophthalmology, 18(1), 1-7.

\section{Copyright holder:}

Rizka Febriya Moestafa (2021)

First publication right:

KESANS : International Journal Health and Science 